\title{
XXIII. On irreversible processes and Planck's theory in relation thereto
}

\section{S.H. Burbury F.R.S.}

To cite this article: S.H. Burbury F.R.S. (1902) XXIII. On irreversible processes and Planck's theory in relation thereto, Philosophical Magazine Series 6, 3:14, 225-240, DOI: 10.1080/14786440209462757

To link to this article: http://dx.doi.org/10.1080/14786440209462757

册 Published online: 08 Jun 2010.

Submit your article to this journal $\pi$

Џ Article views: 2

Q View related articles $\square$

4 Citing articles: 3 View citing articles $\sqsubset$ 
appears also to be reached sooner when the outer cylinder is positive than when it is negative. It follows that as the potential of the cylinder is numerically raised, a voltage is reached at which the positive current overtakes the negative; an event which, however, only happens when the sparking point is nearly reached.

19. These phenomena possibly indicate that when $\mathrm{X} / p$ attains a certain magnitude, positive ions under certain circumstances of impact are capable of generating others by collision, and such an hypothesis would easily account for all the facts, including sparking. But experiments will be undertaken in order, if possible, to throw light on this subject. Another series of experiments are being undertaken to exhibit the difference of behaviour between positively and negatively charged ions generated from, and moving in, hydrogen and carbonic acid gas.

I must take this opportunity of thanking both Professor Townsend, who suggested this research, for his many suggestions, and Professor Turner for his kindness in providing a laboratory, doubtless at great personal inconvenience, in the University Observatory, Oxford, where the experiments were carried out.

XXIII. On Irreversible Processes and Planck's Theory in relation thereto. By S. H. BURBURY, F.R.S.*

DLANCK'S theory is developed in his treatise "Ueber irreversible Strahlungsvorgänge" (Berlin. Sitzungsberichte, 1897, pp. 441-480), and is modified in subsequent papers in detail only. It will be found to depend on some well-known algebraic theorems, and on a certain law of interchange of energy between the parts of a material system.

1. If $u, v$ be two quantities necessarily positive, and which vary subject to the condition that $u+v=2 c$, a constant, the least possible value of $u \log u+v \log v$ is when $u=v$, and the greatest is when either $u=0$ or $v=0$. For we may write $u=c+x, v=c-x, x$ being positive and less than $c$. Then

$$
\begin{gathered}
\frac{d}{d x}(u \log u+v \log v)=\frac{d}{d x}(\overline{c+x} \log \overline{c+x}+\overline{c-x} \log \overline{c-x}) \\
=\log \frac{c+x}{c-x} ;
\end{gathered}
$$

and as this is necessarily positive, the proposition is proved.

- Communicated by the Author.

Phil. Mag. S. 6. Vol. 3. No. 14. Feb. 1902. 
Corollary-If $u^{\prime}, v^{\prime}$ lie between $u$ and $v, u^{\prime} \log u^{\prime}+v^{\prime} \log v^{\prime}$ $<u \log u+v \log n$.

\section{A System of Exchanges.}

2. Let $u, u^{\prime}$ be two essentially positive quantities, for example the energies of two material systems. In time $d t$ let $u$ emit, and $u^{\prime}$ receive, the energy $k u d t$, and let $u^{\prime}$ emit, and $u$ receive, the energy $k^{\prime} u^{\prime} d t, k$ and $k^{\prime}$ being constants.

'Then

$$
\frac{d u}{d t}=k^{\prime} u^{\prime}-k u, \frac{d u^{\prime}}{d t}=k u-k^{\prime} u^{\prime},
$$

and $(a)$ let $\mathrm{S}=u(\log (q u)-1)+u^{\prime}\left(\log \left(q^{\prime} u^{\prime}\right)-1\right)$

$$
\begin{aligned}
\frac{d \mathrm{~S}}{d t} & =\log (q u) \frac{d u}{d t}+\log \left(q^{\prime} u^{\prime}\right) \frac{d u^{\prime}}{d t} \\
& =\left(k^{\prime} u^{\prime}-k u\right) \log \frac{q u}{q^{\prime} u^{\prime}} .
\end{aligned}
$$

If then, $k, k^{\prime}$ being given by the physical conditions, $q, q^{\prime}$ be so chosen that $q / q^{\prime}=k / k, \frac{d \mathrm{~S}}{d t}$ is necessarily negative.

$S$ may be put in other forms. For instance,

(b) let $\mathrm{S}=q u \log (q u)-(1+q u) \log (1+q u)$

$$
\begin{gathered}
+q^{\prime} u^{\prime} \log \left(q^{\prime} u^{\prime}\right)-\left(1+q^{\prime} u^{\prime}\right) \log \left(1+q^{\prime} u^{\prime}\right) \\
\frac{d \mathrm{~S}}{d t}=\left(k^{\prime} u^{\prime}-k u\right) \log \frac{q u}{q^{\prime} u^{\prime}} \frac{1+q^{\prime} u^{\prime}}{1+q u},
\end{gathered}
$$

which is again negative if $q / q^{\prime}=k / k^{\prime}$.

\section{The Physical Application of this Theorem.}

3. A material system is divided into $n$ parts, $a_{1} a_{2} \ldots a_{n}$, and' we assume that the energy, $U$, which the system possesses can be localized, so that at any instant $a_{1} \ldots a_{n}$ possess respectively the energies $u_{1} \ldots u_{n}$, which vary with the time subject to the condition that $u_{1}+\ldots+u_{n}=\Sigma u=\mathrm{U}$.

For the law of this time variation, let us assume that every $a$ emits in time $d t$ a quantity of energy proportional to the corresponding $u$, that is $a_{1}$ emits $k_{1} u_{1} d t$, \&c,

By conservation of energy, what $a_{1}$ emits is received by some one or more of the other parts $a_{2} \ldots a_{n}$. Let us then assume that $k_{12} u_{1} d t$ is received by $a_{2}$

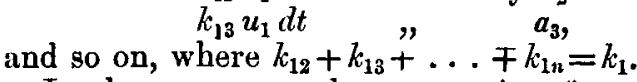

In the same way let $a_{1}$ receive from $a_{2}$ in time $d t$ the energy from $a_{3}$

$$
\begin{array}{ll}
k_{21} u_{2} d t, & \\
k_{31} u_{3} d t, & \text { \&c. }
\end{array}
$$


Then

$$
\begin{aligned}
\frac{d u_{1}}{d t} & =k_{21} u_{2}+k_{91} u_{3}+\ldots+k_{n 1} u_{n} \\
& -k_{12} u_{1}-k_{13} u_{1}-\ldots-k_{1 n} u_{1} .
\end{aligned}
$$

Similarly $\quad \frac{d u_{2}}{d t}=k_{12} u_{1}+k_{32} u_{3}+\ldots+k_{n 2} u_{n}$

and so on.

$$
-k_{21} u_{2}-k_{23} u_{2}-\ldots-k_{2 n_{2}{ }_{2}}
$$

4. Let us now assign to $u_{1} \ldots u_{n}$ respectively the constant positive coefficients $q_{1} \ldots q_{n}$, and form the function

$$
\mathrm{S}=\Sigma(u \log (q u)-u)=\Sigma u \log \frac{q u}{\epsilon}, \text { if } \epsilon=2 \cdot 7182818 \text {, }
$$

Then -.-

$$
\begin{aligned}
& \frac{d \mathrm{~S}}{d t}=\Sigma \log (q u) \frac{d u}{d t} \\
& =\log \left(g_{1} u_{1}\right)\left(k_{21} u_{2}-k_{12} u_{1}\right)+\log \left(q_{2} u_{2}\right)\left(k_{12} u_{1}-k_{21} u_{9}\right) \\
& +\log \left(q_{3} u_{3}\right)\left(k_{18} u_{1}-k_{31} u_{3}\right)+\& c . \\
& +\log \left(q_{1} u_{1}\right)\left(k_{31} u_{3}-k_{18} u_{1}\right)+\log \left(q_{2} u_{2}\right)\left(k_{32} u_{3}-k_{93} u_{9}\right) \\
& +\log \left(q_{5} u_{8}\right)\left(k_{23} u_{2}-k_{32} u_{3}\right)+\& c . \\
& +\log \left(q_{1} u_{1}\right)\left(k_{41} u_{4}-k_{14} u_{1}\right)+\log \left(q_{2} u_{2}\right)\left(k_{42} u_{4}-k_{24} u_{2}\right) \\
& +\& c . \\
& +\log \left(q_{3} u_{8}\right)\left(k_{43} u_{4}-k_{34} u_{3}\right)+\& c . \\
& =\log \frac{q_{1} u_{1}}{q_{2} u_{2}}\left(k_{21} u_{2}-k_{12} u_{1}\right)+\log \frac{q_{1} u_{1}}{q_{3} u_{3}}\left(k_{31} u_{8}-k_{18} u_{1}\right)+\& c . \\
& +\log \frac{q_{2} u_{2}}{q_{3} u_{3}}\left(k_{32} u_{3}-k_{22} u_{2}\right)+\& c
\end{aligned}
$$

and this is necessarily negative, provided that constants $q_{1} \ldots \ldots q_{n}$ exist such that $k_{12} / k_{21}=q_{1} / q_{2}, k_{13} / k_{31}=q_{1} / q_{3}$, \&c , and generally $k_{p r} / k_{r p}=q_{p} / q_{r}$.

If therefore the exchanges of energy between the parts of our system take place according to the above law, the function $\mathbb{S}$ necessarily diminishes with the time, and we may call it the entropy of the system. Further, when $S$ has

reached its minimum, we have:
(1) By conservation of energy $\Sigma \frac{d u}{d t}=0$, and (2) by conservation of entropy $\Sigma \log (q u) \frac{d u}{d t}=0$, and therefore $\log q u$ is an absolute constant, having the same value for every $u$.

Any function whose time-variation has always the same sign until a certain state is reached, and is then zero, may be called an entropy function. It is not necessary that it should

Q 2 
have the logarithmic form. For instance, let $u, v$ be two functions of $t$ which satisfy at every point in a given space the conditions

$$
\frac{d u}{d t}=k v, \quad \text { and } \frac{d v}{d t}=-k^{\prime} u
$$

where $k, k^{\prime}$ are positive constants. These correspond to circular functions. Then if $\mathrm{S}=\iiint u v d x d y d z$ throughout the space in question,

$$
\frac{d \mathrm{~S}}{d t}=\iiint\left(k v^{2}-k^{\prime} u^{2}\right) d x d y d z,
$$

and has always the same sign until on average of the whole space $k \sqrt{v^{2}}=k^{2} u^{2}$. Further, when this state is reached, $\frac{d \mathrm{~S}}{d t}=0$, and $\frac{d^{2} \mathrm{~S}}{d t^{2}}=-4 k k^{\prime} \iiint u v d x d y d z$, and $\mathrm{S}$ retains its minimum value if $\overrightarrow{u v}=0$.

\section{Planck's Theory.}

5. A vacuum space is traversed by an arbitrary system of electromagnetic waves. In this space is a linear electric resonator, or Dipol, whose proper period of vibration corresponds to a wave-length very great in comparison with its own linear dimensions. And it is assumed that its oscillations are damped only by radiation of energy into the surrounding space, and not in any degree by ohmic resistance or other internal dissipative process. Let $f(t)$ denote the mornent at time $t$ of the resonator, $\mathrm{Z}$ the component at time $t$ in the direction of the resonator of the intensity of the electric field at the point where the resonator is, both $f$ and $\mathrm{Z}$ being expressed in absolute electrostatic measure. Then the oscillation of the resonator is given by the equation

$$
\frac{d^{2} f}{d t^{2}}+2 \sigma \nu_{0} \frac{d f}{d t}+4 \pi^{2} v_{0}^{2} f=\frac{3 c^{3} \sigma}{4 \pi^{2} \nu_{0}} \mathrm{Z}, \quad . \quad .
$$

in which $c$ is the velocity of light in vacuo, $\nu_{0}$ the number of oscillations of the resonator per nnit of time, supposing it uninfluenced by any other bodies, and $\sigma$ is the damping, or logarithmic decrement of the amplitude of its oscillations. It is essential to the theory that $\sigma$, and also $\sigma \nu_{0}$, be very small.

6. The vibrations $\mathrm{Z}$ in the surrounding medium may consist of waves of all periods. But expressing $\mathrm{Z}$ in a series of the form

$$
\mathrm{Z}=\int_{0}^{\infty} d \nu \mathrm{C}_{\nu} \cos \left(2 \pi v t-\theta_{\nu}\right)
$$


where $\mathrm{C}_{\nu}$ and $\theta_{\nu}$ are functions of $\nu$, and $\mathrm{C}_{\nu}$ is always positive, Planck obtains (p. 443) for the solution of (1)

in which

$$
f(t)=\frac{3 c^{3}}{1 t ; \pi^{3} \nu_{0}^{2}} \int_{0}^{\infty} d \nu \frac{\mathrm{C}_{\nu}}{\nu} \sin \gamma_{\nu} \cos \left(2 \pi \nu t-\theta_{\nu}-\gamma_{\nu}\right),
$$

$$
\cot \gamma_{\nu}=\frac{\nu_{0}^{2}-v^{2}}{\sigma \nu_{0} v} \pi
$$

Now $\sigma$ is assumed very small. Therefore $\sin \gamma_{\nu}$ is negligible for all values of $v$ except values nearly equal to $\nu_{0}$. Whence also we may write $\cot \gamma_{v}=\frac{\nu_{0}-\nu}{\sigma \nu_{0}} 2 \pi$. Planck concludes that only those waves which have a period very nearly equal to that of the resonator affect it or are affected by it.

Henceforward we will use $\nu$ without the suffix to denote the frequency of the resonator.

7. Planck now distinguishes between rapidly and slowly varying quantities. $Z$ is a rapidly varying quantity, and on the average of a time $\tau$, which though very short contains many complete periods $\frac{1}{v}$, the mean value of $Z$ is zero. But the important thing is not its mean value but the mean value of its square. And $\overline{\mathrm{Z}^{2}}$, if taken on average for two different periods of time, each equal to $\tau$, will generally vary. It belongs to the class of slowly varying quantities. And Planck now defines $\overline{\mathrm{Z}^{2}}$ to be the intensity of the exciting oscillation. In like manuer the mean energy of the resonator is understood to be the mean taken over an interval of time $\tau$ many times greater than the period of the resonator, and other quantities are treated in like manner. The assumption in Planck's theory is fundamental, that we may use these mean values, p. 445 \& p. 457.

8. Let $U$ be the energy of the resonator. Then by known formulæ

in which

$$
\mathrm{U}=\frac{1}{2} \mathrm{~K} f^{2}+\frac{1}{2} \mathrm{~L}\left(\frac{d f}{d t}\right)^{2} \cdot . \cdot \cdot .
$$

$$
\mathrm{K}=\frac{16 \pi^{4} v^{3}}{3 c^{3} \sigma}, \quad \mathrm{L}=\frac{4 \pi^{2} \nu}{3 c^{3} \sigma} \quad . \quad . .
$$

and, $\sigma$ being very small, $\mathrm{K} f^{2}=\mathrm{L}\left(\frac{d f}{d t}\right)^{2}$ on average.

Further, Planck obtains, analysing $\mathrm{Z}$,

$$
\frac{d \mathrm{U}}{d t}+2 \sigma \nu \mathrm{U}=\frac{3 c^{3} \sigma}{16 \pi^{2} v} \mathrm{I}_{\nu},(22) \text { p. } 455,
$$


in which $I$ is the intensity of the vibrations of the surrounding æether whose period is the same as that of the resonator. And $2 \sigma \nu \mathrm{U}$ is the whole energy emitted per unit of time by the resenator having mean energy $U$. This equation, (22) in Planck's notation, is the fundamental equation.

9. Now suppose such a resonator fixed in space, and electromagnetic waves to fall upon it, coming from all directions, and consider these waves at the small distan er from $O$ the centre of the resonator. Taking the axis of the resonator for polar axis, let $\theta, \phi$ be the Houal angular coordinates, so that sin $\theta d \theta d \phi$ defines the solid angle $d \Omega$ at $O$ in direction $\boldsymbol{\theta} \phi$.

Let us consider these waves divided into separate waves, each having front $r^{2} d \Omega$. I tet $\mathbf{K}$ denote the intensity of the vibrations of the ware. Consider an element of area $d s=r^{2} d \Omega$ on the surface of the $r$ sphere, and an element $d \sigma$ at the centre perpendicular to the radius to $d s$. Then the energy which in time $d t$ passes from $d s$ to $d \sigma$ is $d t \frac{d s d \sigma}{r^{2}} \mathbf{K}$ (p. 456), that is $d t d \sigma . d \Omega \mathrm{K}$. It follows that the energy per unit of volume at the centre due to the wave is the lasti expression divided by $c d t d \sigma$, that is $\frac{\mathbf{K}}{\mathbf{c}} d \Omega$, and the whole energy per unit of volume at the centre is, if $\mathbf{K}$ is constant for all positions of $d \Omega, \frac{4 \pi \mathbf{K}}{c}$.

10. The vibrations are in the plane of the wave. But the polarization may have any direction in that plane. There are then, continues Planck, in that plane two mutually perpendicular directions in which the vibrations have intensity respectively greater and less than in any other. These are called the principal directions, and the intensities of vibration in them, which shall be denoted by $\mathbf{K}, \mathbf{K}^{\prime}$, are the principal intensities.

We might, however, resolve the vibrations in any other two mutually perpendicular directions in the plane of the wave, making with the principal directions the angles $\omega$ and $\frac{\pi}{\omega}+\omega$ respectively If $\mathbf{K}_{1}, \mathbf{K}_{2}$ denote the intensities of these resolved vibrations respectively,

$$
\begin{aligned}
& \mathbf{K}_{1}=\mathbf{K} \sin ^{2} \omega+\mathbf{K}^{\prime} \cos ^{2} \omega, \\
& \mathbf{K}_{2}=\mathbf{K} \cos ^{2} \omega+\mathbf{K}^{\prime} \sin ^{2} \omega,
\end{aligned}
$$

and $K_{1}+K_{2}=K+K^{\prime}$ whatever $\omega$ may be; also $K_{1}$ and $K_{2}$ lie between $K$ and $\mathbf{K}^{\prime}$. 
Let now the plane of the paper be that of the wave, and let the dotted line $\mathrm{O} z$ be the direction of the resonator axis which makes the angle $\theta$ with the normal.

Let $\mathrm{OP}$ be the projection of $\mathrm{O} z$ on the plane, $\mathrm{OR}$ a line in

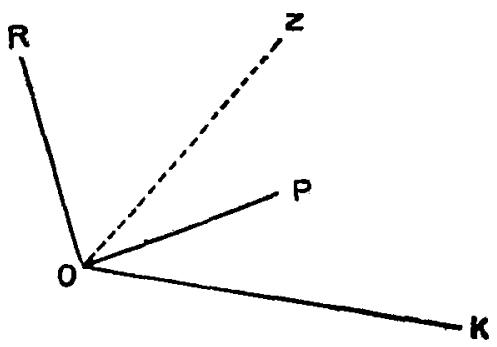

the plane perpendicular to $\mathrm{OP}$, and therefore to $\mathrm{Oz}$. Also let OK denote the principal direction corresponding to the intensity $\mathbf{K}$.

Then the angle $\mathrm{KOP}=\omega$

$$
\text { and } \mathrm{PO} z=\frac{\pi}{2}-\theta \text {. }
$$

$\mathbf{K}_{1}$ is the intensity of vibration in $\mathrm{OR}$,

$\mathbf{k}_{\mathbf{q}} \quad " \quad$ " OP.

The electric force due to the vibrations $K_{2}$ in $O P$ is perpendicular to $O P$, that is in $O R$, and is therefore perpendicular to $0 z$. Therefore the vibrations $\mathbf{K}_{2}$ have no electric force in $\mathrm{Oz}$. They do not affect, and are not affected by, the resonator. If $\partial \mathbf{K}$ denotes for any $\mathbf{K}$ the change in it due to incidence on the resonator, $\partial \mathbf{K}_{2}=0$.

On the other hand the vibrations in $\mathrm{OR}$, whose intensity is $\mathrm{K}_{1}$, have electric force in $\mathrm{OP}$, which makes the angle $\frac{\pi}{2}-\theta$ with the axis. 'Therefore a part of the energy of these vibrations is absorbed by the resonator, and lost to the wave. And this part is in time $d t, \frac{3 c^{2} \sigma}{4 \pi v} \mathrm{~K}_{\mathrm{l}} \sin ^{2} \theta d t d \Omega$ (p. 461, equation (36)).

It should be noted that

$$
\begin{aligned}
3 c^{2} \sigma & =\frac{3}{4 \pi \nu} \frac{c^{2}}{4 \pi} \nu^{2} \sigma \nu \\
& =\frac{3}{4 \pi} \lambda^{2} \sigma \nu
\end{aligned}
$$

where $\lambda$ is the wave-length. Now $\sigma \nu$ has been assumed to 

be very small. Therefore $\frac{3 c^{2} \sigma}{4 \pi v}$ is a quantity whose square
may be neglected.

The result stated above for the absorbed energy involves a relation between the magnitude of the elementary area $r^{2} d \Omega$, that is the section of the wave which we are considering, and the cross section of the resonator. For the quantity of energy absorbed by the resonator will depend on the cross section, so therefore must $d \Omega$. I understand Planck to state this, p. 461.

11. Again, the energy emitted by the resonator per unit of time is $2 \sigma \nu \mathrm{U}$ (art. 8). It is emitted uniformly in all directions, making with the axis angles between $\theta$ and $\theta+d \theta$. But the energy emitted in such directions is proportional to $\sin ^{2} \theta$. Therefore the energy emitted in any direction $d \Omega$ is per unit of time

$$
\frac{2 \sigma \nu \mathrm{U} \sin ^{2} \theta d \Omega}{\int \sin ^{2} \theta d \Omega}=\frac{3 \sigma \nu \mathrm{U}}{4 \pi} \sin ^{2} \theta d \Omega,
$$

because $j \sin ^{2} \theta d \Omega=\frac{8 \pi}{3}$.

12. The energy thus emitted by the resonator is absorbed by the waves of the same period $\frac{1}{v}$. It follows that if $f \mathbf{K}_{1} d \Omega d t$ denotes the energy of the wave of period $\frac{1}{v}$ which passes the elementary area $r^{2} d \Omega$ in time $d t$,

$$
\begin{aligned}
\frac{d}{d t}\left(f \mathbf{K}_{1}\right)=f \frac{d \mathrm{~K}_{1}}{d t} & =-\frac{3 c^{2} \sigma}{4 \pi \nu} \mathbf{K}_{1} \sin ^{2} \theta+\frac{3 \sigma \nu}{4 \pi} \mathrm{U} \sin ^{2} \theta \\
& =\frac{3 c^{2} \sigma}{4 \pi \nu}\left(\frac{\nu^{2} \mathrm{U}}{c^{2}}-\mathbf{K}_{1}\right) \sin ^{2} \theta .
\end{aligned}
$$

But

$$
\frac{d \mathrm{U}}{d t}=\frac{3 c^{2} \sigma}{4 \pi \nu}\left(\mathbf{K}_{1}-\frac{\nu^{2} \mathrm{U}}{c^{2}}\right) \sin ^{2} \theta \text {. }
$$

Therefore

$$
\frac{d\left(f \mathbf{K}_{1}\right)}{d t}+\frac{d \mathrm{U}}{d t}=0
$$

expresses the conservation of energy.

13. The conditions of the transfer of energy between resonator and wave are precisely the conditions necessary for application of the theorem of art. 3. Each system emits to the other per unit of time an amount of energy proportional to the energy which the emitting system has for the time being. Further, the ratio $k_{12} / k_{21}$ of art. 2 is here $c^{2} / \nu^{2}$. We expect then to find that an entropy function exists. 
The silectromagnetic Entropy.

14. According to Planck's definition, the entropy, $S$, of the resonator whose energy is $\mathrm{U}$, is

$$
\mathrm{S}=-\frac{\mathrm{U}}{a \nu}\left(\log _{l, \nu}^{\mathrm{U}}-1\right)
$$

where $a$ and $b$ are constants. It is indepondent of the entropy of the incident waves.

Again, if $s$ denote the entropy per unit of volume of a monochromatic wave $d \Omega$ of intensity $\mathbf{K}$, we have by analogy to art. $9, s=d \Omega \frac{\mathrm{L}}{\mathrm{c}}$, where

$$
\mathrm{L}=-\frac{\mathrm{K}}{a \nu}\left(\log \frac{e^{2} \mathrm{~K}}{b \nu^{3}}-1\right),
$$

which has a determinate value at every point. The actual entropy of any given volume of the wave thronghout which $\boldsymbol{K}$ is constant is, or is proportional to, the last expression multiplied by the given volume.

15. Up to this point I have closely followed Planck. I now deviate somewhat from his method. Let $f \mathbf{K} d \Omega d t$ denote as before the energy of the wave of intensity $\mathbf{K}$ which passes the elementary area $r^{2} d \Omega$ in time $d t$. T'hen $f$ is proportional to the volume of the ware in question. And let us define the entropy of that same wave as follows:

$$
s=-\frac{1}{a v} f \mathbf{K}\left(\log \frac{c^{2} \mathbf{K}}{b v^{3}}-1\right) d \Omega d t,
$$

whence

$$
\frac{d s}{d t}=-\frac{1}{a \nu} \log \frac{c^{2} \mathbf{K}}{b \boldsymbol{v}^{3}} \frac{d(f \mathbf{K})}{d t} d \Omega .
$$

Then we have for an unpolarized wave

$$
\frac{d}{d t}(\mathrm{~S}+s)=-\frac{1}{a v} \log \frac{\mathrm{U}}{b v} \frac{d \mathrm{U}}{d t}-\frac{1}{a v} \log \frac{c^{2} \mathrm{~K}}{b v^{3}} \frac{d(f \mathrm{~K})}{d t} .
$$

But

$$
\frac{d U}{d t}=\frac{3 c^{2} \sigma}{4 \pi \nu}\left(\mathrm{K}-\frac{\nu^{2} \mathrm{U}}{c^{2}}\right) \sin ^{2} \theta,
$$

and

$$
\frac{d(f \mathbf{K})}{d t}=\frac{3 c^{2} \sigma}{4 \pi \nu}\left(\frac{\nu^{2} \mathrm{U}}{c^{2}}-\mathbf{K}\right) \sin ^{2} \theta .
$$

Therefore

$$
\frac{d(\mathrm{~S}+s)}{d t}=-\frac{1}{a \nu} \frac{3 c^{2} \sigma}{4 \pi v} \sin ^{2} \theta\left(\frac{\nu^{2} \mathrm{U}}{c^{2}}-\mathbf{K}\right) \log \frac{c^{2} \mathbf{K}}{v^{2} \mathrm{U}},
$$


which, owing to the minus sign prefixed, is necessarily positive if not zero, and then only zero when $\mathbf{K}=\frac{\nu^{2} \mathrm{U}}{c^{2}}$ for every wave and resonator.

\section{A Polarized Wave.}

16. We cannot in calculating entropy, as we did in calculating energy, use $\mathbf{K}_{1}$ and $\mathbf{K}_{2}$ instead of the principal intensities $\mathbf{K}, \mathbf{K}^{\prime}$. For $\mathbf{K}_{1}$ and $\mathbf{B}_{2}$ lie between $\mathbf{K}$ and $\mathbf{K}^{\prime}$.

Therefore by art. 1

$$
-\frac{\mathrm{K}_{1}}{a \nu}\left(\log \frac{c^{2} \mathbf{K}_{1}}{b \nu^{3}}-1\right)-\frac{\mathrm{K}_{2}}{a \nu}\left(\log \frac{c^{2} \mathbf{K}_{2}}{b \nu^{3}}-1\right)
$$

is greater than

$$
-\frac{\mathbf{K}}{a v}\left(\log \frac{c^{2} \mathbf{K}}{b v^{3}}-1\right)-\frac{\mathbf{K}^{\prime}}{a v}\left(\log \frac{c^{2} \mathbf{K}^{\prime}}{b v^{3}}-1\right) .
$$

Let us then define

$$
s=-\frac{1}{a v} f \mathbf{K}\left(\log \frac{c^{2} \mathbf{K}}{b \nu^{3}}-1\right)-\frac{1}{a v} f \mathbf{K}^{\prime}\left(\log \frac{c^{2} \mathbf{K}^{\prime}}{b \nu^{3}}-1\right)
$$

to be the actual entropy of the wave above defined before incidence. And let

$$
s_{1}=-\frac{1}{a \nu} f \mathbf{K}_{1}\left(\log \frac{c^{2} \mathbf{K}_{1}}{b \nu^{3}}-1\right)-\frac{1}{a \nu} f \mathbf{K}_{2}\left(\log \frac{c^{2} \mathbf{K}_{2}}{b \nu^{3}}-1\right)
$$

denote the hypothetical entropy of the wave before incidence. It is what the entropy would be if $\mathbf{K}_{1}$ and $\mathbf{K}_{2}$ were the principal intensities.

After incidence let $s$ become $s^{\prime}$, and $s_{1}$ become $s_{1}^{\prime}$. And similarly the entropy of the resonator shall be $\mathbf{S}$ before and $\mathbf{S}^{\prime}$ after, incidence.

Now, as we have seen, $\frac{d}{d t}\left(f^{\prime} \mathbf{K}_{2}\right)=0 . \quad$ But

$$
\frac{d}{d t}\left(f \mathbf{K}_{1}\right)=-\frac{3 c^{2} \sigma}{4 \pi v}\left(\mathbf{K}_{1}-\frac{\nu^{2} \mathrm{U}}{c^{2}}\right) \sin ^{2} \theta .
$$

We will, following Planck, denote by $\mathbf{K}_{\mathbf{3}}$ what $\mathbf{K}_{\mathbf{1}}$ becomes after incidence. We have then

$$
\begin{aligned}
s_{1}^{\prime}-s_{1} & =-\frac{1}{a \nu} \log \frac{c^{2} \mathbf{K}_{1}}{b v_{3}} \frac{d\left(f \mathbf{K}_{1}\right)}{d t} d t \\
& =+\frac{1}{a \nu} \log \frac{c^{2} \mathbf{K}_{1}}{b \nu^{3}} \frac{3 c^{2} \sigma}{4 \pi \nu}\left(\mathbf{K}_{1}-\frac{\nu^{2} U}{c^{2}}\right) \sin ^{2} \theta d t
\end{aligned}
$$


Also

$$
\begin{aligned}
\mathrm{S}^{\prime}-\mathrm{S} & =-\frac{1}{a \nu} \log \frac{\mathrm{U}}{b \nu} \frac{d \mathrm{U}}{d t} d t \\
& =+\frac{1}{u \nu} \log \frac{\mathrm{U}}{b_{\nu}} \frac{3 c^{2} \sigma}{4 \pi \nu}\left(\frac{\nu^{2} \mathrm{U}}{c^{z}}-\mathbf{K}_{1}\right) \sin ^{2} \theta d t .
\end{aligned}
$$

Therefore

$$
s_{1}^{\prime}+\mathrm{S}^{\prime}-\left(s_{1}+\mathrm{S}\right)=-\frac{1}{a \nu} \frac{3 c^{2} \sigma}{4 \pi \nu} \sin ^{2} \theta\left(\frac{\nu^{2} \mathrm{U}}{c^{2}}-\mathbf{K}_{1}\right) \log \frac{c^{2} \mathrm{~K}_{1}}{\nu^{2} \tilde{U}^{\prime}} d t,
$$

which is necessarily positive owing to the minus sign prefixed.

But $s_{1}>s$ by art. 1 .

Therefore, a fortiori, $s_{1}{ }^{\prime}+\mathrm{S}^{\prime}>s+\mathrm{S}$; or the entropy of the resonator plus the hypothetical entropy of the wave after incidence is greater than the sum of the actual entropies of wave and resonator before incidence.

17. But also $s_{1}^{\prime}>s^{\prime}$ by art. 1 .

It does not therefore yet follow that $s^{\prime}+\mathrm{S}^{\prime}>s+\mathrm{S}$, which is the proposition we wish to prove.

To make the proof complete at this point it is necessary to assume that $\mathbf{K}_{2}$ and $\mathbf{K}_{3}$ are after incidence the principal intensities; and therefore $s_{1}^{\prime}=s^{\prime}$. And this Planck asserts to be the case (p. 468).

$\mathrm{He}$ does not, however, explain the grounds for this assertion. The principal directions before incidence are independent of the direction $\mathrm{O} z$ (see figure) of the resonator axis. They cannot therefore generally be $O P, O K$, which are determined by the direction $\mathrm{O} z$. Therefore generally $\mathbf{K}_{2}$ in $O P$ is neither the greatest nor the least intensity. By the incidence on the resonator the intensity $\underline{K}_{1}$ in $\mathrm{OR}$ is altered by a very small quantity proportional to $\frac{3 c^{2} \sigma}{4 \pi \nu}$. This cannot generally have the effect of making $\mathbf{K}_{2}$ either the greatest or the least intensity.

18. The proof can, however, as seems to me, be completed as follows:-For any given wave-front, all directions of the axis $\mathrm{O} z$ of the resonator are equally probable. Therefore on average of all waves and resonators with the same $\nu$,

And now

$$
\left(s_{1}-s\right)=\left(s_{1}^{\prime}-s^{\prime}\right) \text {. }
$$

$$
\begin{aligned}
s^{\prime}+\mathrm{S}^{\prime}-(s+\mathrm{S}) & =s_{1}^{\prime}+\mathrm{S}^{\prime}-\left(s_{1}+\mathrm{S}\right)+\left(s_{1}-s\right)-\left(s_{1}^{\prime}-s^{\prime}\right) . \\
& =s_{1}^{\prime}+\mathrm{S}^{\prime}-\left(s_{1}+\mathrm{S}\right), \text { on average. }
\end{aligned}
$$

And this we have proved to be positive. 
It follows that, on average of all waves and resonators having the same $\nu$, the entropy must increase until a state is reached, the stationary state, in which

$$
\mathbf{K}_{1}=\mathbf{K}_{2}=\mathbf{K}=\mathbf{K}^{\prime}=\frac{\nu^{2} \mathbf{U}}{c^{2}} .
$$

Another difficulty that $I$ find is as follows:-Assuming that $\mathbf{K}_{2}$ and $\mathbf{K}_{\mathbf{3}}$ are the principal intensities after incidence, the argument proceeds in Planck's own words as follows:"wird der Resonator von einem irgendwie polarisirten Strahlenbündel getroffen, dessen Energiestrahlung die Haupt. intensitäten $\mathbf{K}$ und $\mathbf{K}^{\prime}$, und dessen Entropiestrahlung daher die Intensität $L+L^{\prime}$ besitzt. Dieses Strahlenbündel lässt in der Zeit $d \iota$ die Entropie

$$
\text { " }\left(\mathrm{L}+\mathrm{L}^{\prime}\right) d t \frac{3 c^{2} \sigma}{4 \pi \nu} d \Omega,
$$

auf den Resonator fallen, und dadurch wird auf dieser Seite der nämliche Entropiebetrag dem Felde entzogen. Auf der anderen Seite geht vom Resonator ein in bestimmter Weise polarisirtes Strahlenbündel aus dessen Energiestrahlung die Hauptintensitäten $\mathbf{K}_{2}$ und $\mathbf{K}_{3}$, und dessen Entropiestrahlung daher die entsprechende Intensitäten $L_{2}$ und $L_{3}$ besitzt. Dadurch wird dem umgehenden Felde die Entropie

$$
\text { " }\left(\mathrm{L}_{2}+\mathrm{L}_{3}\right) d t \frac{3 c^{2} \sigma}{4 \pi v} d \Omega,
$$

zugeführt. Im ganzen beträgt also die in der Zeit $d t$ eingetretene Entropieänderung des den Resonator umgebenden Feldes

$$
\text { “dt } \frac{3 c^{2} \sigma}{4 \pi \nu} \dot{j}\left(\mathrm{~L}_{2}+\mathrm{L}_{3}-\mathrm{L}-\mathrm{L}^{\prime}\right) d \Omega . \quad(46) "
$$

And to this has to be added the change of entropy of the resonator itself. And Planck thence proves that the sum of the entropies is increased.

The difficulty in this reasoning is in the introduction of the factor $\frac{3 c^{2} \sigma}{4 \pi \nu}$. If $\partial$ denotes for any function the change due to incidence on the resonator, or the difference between the values of the function before and after incidence, then the quantity in brackets in the expression (46) is equivalent (on Planck's assumption that $\mathbf{K}_{\mathbf{2}}$ and $\mathbf{K}_{\mathbf{3}}$ are principal intensities) to $\partial\left(\mathrm{L}+\mathrm{L}^{\prime}\right)$, that is to

$$
-\frac{1}{u \nu}\left\{\log \frac{c^{2} \mathbf{K}}{b \nu^{3}} \partial \mathbf{K}+\log \frac{c^{2} \mathbf{K}^{\prime}}{b \nu^{3}} \partial \mathbf{K}^{\prime}\right\} \text {. }
$$


Further, from the equations

$$
\begin{aligned}
& \mathbf{K}_{1}=\mathbf{K} \sin ^{2} \omega+\mathbf{K}^{\prime} \cos ^{2} \omega, \\
& \mathbf{K}_{2}=\mathbf{K} \cos ^{2} \omega+\mathbf{K}^{\prime} \sin ^{2} \boldsymbol{\omega},
\end{aligned}
$$

$\mathbf{K}$ and $\mathbf{K}^{\prime}$ may be expressed as linear. functions of $\mathbf{K}_{1}$ and $\mathbf{K}_{2}$. And since $\partial \mathbf{K}_{2}=0$, and $\partial \mathbf{K}_{1}$ is proportional to $\frac{3 c^{2} \sigma}{4 \pi v}$ by $(36)$, it follows that $\partial \mathbf{K}$ and $\partial \mathbb{K}^{\prime}$, and therefore also $\partial\left(L+L^{\prime}\right)$, and therefore also the quantity in brackets in (46), is proportional to $\frac{\partial \vec{\partial} c^{2} \sigma}{4 \pi v}$. The factor $\frac{3 c^{2} \sigma}{4 \pi \nu}$ is then not wanted outside of the bracket, and has no right to be there.

It is true that since $\mathrm{L}^{\prime} c$ denotes entropy per unit of volume, the quantity in brackets requires some factor to make it express an actual amount of entropy. But such factor must be independent of $\sigma$, and therefore cannot be $\frac{3 c^{2} \sigma}{4 \pi \nu}$. The above are the difficulties which I have found. Very probably they are capable of explanation.

\section{On Planck's Theory in relation to the Distribution of Energy in the Spectrum.}

19. Planck has given no account of interchanges of energy between systems of different vibration periods. His method is in fact based on the assumption or proof (art. 6) that waves of different period from that of a resonator pass the resonator unatfected, so that no interchange of energy takes place. This, however, is not quite rigorous. If the difference of periods, though not zero, be very small, some very small interchange of energy between the wave and the resonator will consistently with the equation of p. 443 take place. We may admit, therefore, that if all values of $v$ are represented, $\nu$ varying continuously between different systems, energy will pass indirectly from systems of period $\frac{1}{v}$ to systems of period $\frac{1}{v}$, though the rate of interchange will be much slower than between systems of the same period.

20. Now Planck does not investigate the law of these slow interchanges. He assumes that an entropy function exists for them, and that it is precisely the same function (but with variable $\nu$ ) which has been defined above for systems having the same period. That may be true, but it cannot, I think, be accepted as an axiom. It seems to me that this branch of the subject requires further elucidation. 
21. According to Planck, if the entropy has the general form $p \mathrm{U} \log \frac{q \mathrm{U}}{\epsilon}$ for the resonator, and $p \mathbf{K} \log \frac{q^{\prime} \mathbf{K}}{\epsilon}$ for the wave, we have

$$
p=-\frac{1}{a v}, \quad q=\frac{1}{b v}, \quad q^{\prime}=\frac{c^{2}}{v^{2}} \frac{1}{b v},
$$

where $v$ is now a variable. As he himself explains, the constants $p, q, q^{\prime}$ are not determined by the analysis (which appears to determine the ratio $q^{\prime} / q$ but no more), but are chosen to make the results of analysis agree with those of experiment.

22. For any infinitely small "virtual" change of state the variation of the total entropy must in the stationary state vanish. The "virtual" change of state supposed is that a small quantity of energy proportional to $\partial \mathbf{R}_{1}$ is added to one system of period $\frac{1}{v_{1}}$, and another small quantity proportional to $\partial K_{2}$ is added to another system of period $\frac{1}{v_{2}}$, ceteris paribus. Now a "virtual" change-if there is any virtue in the term - means a change consistent with the conditions of our system. In this supposition, therefore, we have definitely thrown overboard the restriction that energy can only be interchanged between systems of the same period.

However, given that the above change takes place, we have by conservation of energy

$$
\partial K_{1}+\partial K_{2}=0 \text {. }
$$

And by conservation of entropy in the stationary state,

$$
p_{1} \log \left(q_{1} \mathbf{K}_{1}\right) \partial \mathbf{K}_{1}+p_{2} \log \left(q_{2} \mathbf{K}_{2}\right) \partial \mathbf{K}_{2}=0 .
$$

From which it follows that in the stationary state $p \log (q \mathbf{K})$ is an absolute constant, independent of $v$.

Assume

$$
p \log (g \mathbf{K})=-\frac{1}{\theta}
$$

whence

$$
\mathbf{K}=\frac{1}{q} \epsilon^{-\frac{1}{p \theta}}
$$

Instead of $\nu$ Planck now introduces the new rariable $\lambda$, the wave-length, by the formula

$$
\begin{aligned}
\lambda & =\frac{c}{\nu}, \\
d \nu & =-\frac{c}{\lambda^{2}} \cdot d \lambda .
\end{aligned}
$$


$\Lambda$ nd thence using his own form for $p$ and $q$, namely $p=\frac{1}{a v}$, $q=\frac{1}{b v^{3}}$, he obtains for the intensity $\mathrm{E}_{\lambda}$, of the wave-length $\lambda$,

$$
\mathrm{E}_{\lambda}=\frac{2 b c^{2}}{\lambda^{5}} \boldsymbol{\epsilon}^{-\frac{a c}{\lambda \theta}}
$$

which is Wien's law. And this at the date of Planok's paper now under discussion was considered to be sufficiently. accurate.

23. Later researches of Beckmann, Pringsheim, and others throw doubt on the accuracy of Wien's law. It becomes then necessary, in order to make the results of Planck's analysis agree with experiments, either to vary the constants $p, q, q^{\prime}$, or else to vary the form of the function $\mathrm{S}$. And this Planck does in his paper "Ueber irreversible Strahlungsvorgänge" (Preuss. Akad.Wiss. Berlin, Sitzungsherichte, xxv. pp. 544-555, May 9, 1901). He there, without altering the general theory as developed in the former treatise, assume: for the entropy of the resonator

$$
\mathrm{S}=k\left\{\left(1+\frac{\mathrm{U}}{h v}\right) \log \left(1+\frac{\mathrm{U}}{h v}\right)-\frac{\mathrm{U}}{h \nu} \log \frac{\mathrm{U}}{h v}\right\},
$$

where $k$ and $l$ are constants, and obtains results in accordance with the later experiments.

\section{Is Planck's a true irreversible process?}

24. According to the theorems of art. 2, every interchange of energy between the parts of a material system, if such interchanges are made in accordance with the law there assumed, is an irreversible process, just as, and for the same reason that, interchanges of heat between hot and cold bodies are irreversible. Now according to Planck the interchanges of energy between different parts of his system do take place according to the law assumed in art. 2 . The process, if it exists in nature, is therefore an irreversible process.

But the existence of such a machine as Planck's resonator cannot be proved by experiment. For any actual electric vibration such as he supposes must be accompanied by dissipation of energy. It may be that ather, the universal solvent of mathematical difficulties, supplies us with the instrument required. But if not, Planck's process is non-existent. And can a non-existent process be irreversible?

25. It seems to me that if at any instant all the velocities in Planck's problem were reversed, the system would not retrace 
its course, executing in the reverse direction all the changes through which it has passed. In this respect Planck's problem differs toto colo from that of Boltzmann's $\mathrm{H}$ theorem, where we have a process, consisting of a number of separately reversible processes, supposed to be irreversible in the aggregate.

XXIV. Note on a Paper by Prof. Fleming, F.R.S., and Mr. Ashton, entitled "On a Model which Imitates the Behaviour of Dielectrics." By John Buchanan, D.Se. (Lond.)*.

TNDER the above title there has appeared in the Phil. Mag. for August 1901 a description of a very ingenious model invented by the authors of the paper. The diagrams, obtained by help of the model, which illustrate the paper are exceedingly interesting and suggestive.

There are some points in the theory of the action of the model, and of the behaviour of the dielectric in a condenser, which may be deemed worth attention.

1. The action of the model clearly depends an the viscosity of a liquid. The diagrams show by their form the very interesting fact that the motion of the pencil which traced them approximated closely to what may be expressed by the term "motion of a viscous fluid by diffusion" $\neq$.

In other words, the displacement curves obtained from the model, and their derived velocity curves, are of the same form as the graphs of certain solutions of Fourier's well-known equation

$$
\frac{d v}{d t}=\mathrm{K} \frac{d^{2} v}{d x^{2}} . \quad . . . . .
$$

For comparison with figs. I. \& III. (Pl. V.) respectively, of Prof. Fleming and Mr. Ashton's paper, I give here figs. 1 and 2, which are the respective graphs of equations (2) and (3) below. In these solutions of (1) $v$ denotes the displacement, $t$ the time, $x$ the distance from the origin at which exists the motion under consideration, and $K$ denotes the "diffusivity."

Without the guidance afforded by some such theory as is here given, it would be quite impossible to discover from experiment such a law of displacement as is expressed by (2) for instance, or of velocity as expressed by (3). Thus, the

* Communicated by the Physical Society : read November 8, 1901.

$+C f$. Lord Kelvin's Math. and Phys. Papers, vol, iii. art. xcviii. p. 433. 